\title{
Error Analysis for Direct Linear Integral Equation Methods*
}

\author{
By James L. Phillips
}

\begin{abstract}
An error analysis of projection methods for solving linear integral equations of the second kind is presented. The relationships between several direct methods for solving integral equations are examined. It is shown that the error analysis given is applicable to other methods, including a modified Nyström method and certain degenerate kernel methods.
\end{abstract}

1. Introduction. Consider a linear integral equation of the second kind,

$$
\lambda x(s)-\int_{a}^{b} k(s, t) x(t) d t=y(s), \quad a \leqq s \leqq b,
$$

or in operator form

$$
(\lambda I-K) x=y .
$$

The equation is assumed to be in the Banach space $C[a, b]$ of continuous functions on $[a, b]$ normed with the sup norm. We further assume $K: C[a, b] \rightarrow C[a, b]$ is a compact operator and that $\lambda \neq 0$ is not an eigenvalue of $K$. Then the equation has a unique solution $x^{*}(s)$ for any given $y \in C[a, b]$.

When a projection method is used to find an approximate solution to the above equation, (1.2) is replaced by

$$
\left(\lambda I-P_{n} K\right) x_{n}=P_{n} y .
$$

Here $P_{n}$ is a projection (a linear, idempotent) operator from $C[a, b]$ onto a finitedimensional subspace $S_{n}$ of $C[a, b]$. Let $M$ denote a finite-dimensional subspace of the space of continuous linear functionals on $C[a, b]$, and set

$$
M_{\perp}=\{f \in C[a, b]: \mu(f)=0 \text { for each } \mu \in M\} .
$$

A projection $P_{n}$ with range $S_{n}$ and kernel $M_{\perp}$ is determined if and only if $S_{n} \cap M_{\perp}=$ \{0\}. If $\left\{\mu_{i}\right\}_{1}^{n}$ is a basis of $M$ and $\left\{y_{i}\right\}_{1}^{n}$ is a basis of $S_{n}$ such that

$$
\mu_{i}\left(y_{i}\right)=\delta_{i i}, \quad i, j=1, \cdots, n,
$$

$P_{n}$ is defined by

$$
\left(P_{n} f\right)(s)=\sum_{i} \mu_{i}(f) y_{i}(s), \quad f \in C[a, b] .
$$

Received August 9, 1972, revised November 20, 1972.

AMS (MOS) subject classifications (1970). Primary 45L10, 65R05; Secondary 41A10, 41A15, 45A05, 45B05, 65D05.

Key words and phrases. Projection methods, Galerkin method, Nyström method, degenerate kernel methods, Chebyshev expansion, finite-rank operators.

* This work was supported in part by the National Science Foundation under Grant GJ-31827. 
When a solution $x_{n}$ of (1.3) exists, it can be found by choosing a basis $\left\{\mu_{i}\right\}$ of $M$ and a basis $\left\{u_{i}\right\}$ of $S_{n}$. Then

$$
x_{n}(s)=\sum_{i} c_{i} u_{i}(s)
$$

is determined by solving the linear system

$$
\sum_{i}\left[\lambda \mu_{i}\left(u_{i}\right)-\mu_{i}\left(K u_{i}\right)\right] c_{i}=\mu_{i}(y), \quad i=1, \cdots, n,
$$

for $\left\{c_{i}\right\}$.

Projection methods are examined as a special case of more general approximation methods in [9]. A further analysis with numerical examples is given in [13]. See also [8]. In the next section, we extend the analysis of [13] to include the error due to the use of quadrature in (1.7). A method for constructing quadrature rules for use with projection methods and two examples are given in Section 3. A general class of finite-rank operator methods which includes Nyström and degenerate kernel methods is examined in Section 4, and the analysis of Section 2 is shown to apply to these methods.

2. Error Analysis of Projection Methods. The analysis presented here is motivated by two particular projection methods, collocation and Galerkin's method. The method of collocation is based on projection by interpolation. Thus, the $\mu_{i}$ in (1.5) are given by

$$
\mu_{i}(f)=f\left(t_{i}\right), \quad t_{i} \in[a, b],
$$

and the system (1.7) becomes

$$
\sum_{i}\left[\lambda u_{j}\left(t_{i}\right)-\int_{a}^{b} k\left(t_{i}, t\right) u_{j}(t) d t\right] c_{i}=y\left(t_{i}\right), \quad i=1, \cdots, n .
$$

Orthogonal or Fourier projection is used in Galerkin's method. If the $\left\{u_{i}\right\}$ satisfy

$$
\int_{a}^{b} w(t) u_{i}(t) u_{j}(t) d t=\delta_{i i}
$$

for some $w(t) \geqq 0$, the functionals in (1.5) are given by

$$
\mu_{i}(f)=\int_{a}^{b} w(t) u_{i}(t) f(t) d t .
$$

In this case, (1.7) becomes

$$
\begin{aligned}
& \sum_{i}\left[\lambda \delta_{i i}-\int_{a}^{b} w(s) u_{i}(s) \int_{a}^{b} k(s, t) u_{j}(t) d t d s\right] c_{i} \\
&=\int_{a}^{b} w(s) u_{i}(s) y(s) d s, \quad i=1, \cdots, n .
\end{aligned}
$$

When the integrals in (2.2) or the inner integrals in (2.5) are replaced by a quadrature rule, the approximating equation being solved is not (1.3), but an equation of the form

$$
\left(\lambda I-P_{n} K_{m}\right) x_{n m}=P_{n} y .
$$


Suppose $\left\{P_{n}\right\}$ and $\left\{K_{m}\right\}$ are sequences of operators such that

$$
\left\|K-K_{m}\right\| \rightarrow 0 \text { as } m \rightarrow \infty
$$

and

$$
\left\|K-P_{n} K\right\| \rightarrow 0 \text { as } n \rightarrow \infty .
$$

A technique for constructing operators which satisfy (2.7) is discussed in the next section. Sufficient conditions on $K$ and $P_{n}$ for (2.8) are given in [13].

THEOREM 2.1. Conditions (2.7) and (2.8) imply

$$
\lim _{n \rightarrow \infty} \lim _{m \rightarrow \infty}\left\|K-P_{n} K_{m}\right\|=0 .
$$

Moreover, if $\left\{\left\|P_{n}\right\|\right\}$ is uniformly bounded,

$$
\lim _{n, m \rightarrow \infty}\left\|K-P_{n} K_{m}\right\|=0 .
$$

Hence, for all $n$ and $m$ such that

$$
\left\|K-P_{n} K_{m}\right\|\left\|(\lambda I-K)^{-1}\right\|<1,
$$

a unique solution $x_{n m}$ of (2.6) exists. Furthermore,

$$
\begin{aligned}
\left\|x^{*}-x_{n m}\right\| \leqq & \left\|\left(\lambda I-P_{n} K_{m}\right)^{-1}\right\| \\
& \cdot\left\{|\lambda|\left(1+\left\|P_{n}\right\|\right) \operatorname{dist}\left(x^{*} ; S_{n}\right)+\left\|P_{n}\right\|\left\|\left(K-K_{m}\right) x^{*}\right\|\right\},
\end{aligned}
$$

where dist $\left(x^{*} ; S_{n}\right)=\inf _{o \in S_{n}}\left\|x^{*}-g\right\|$.

Proof. For each $n$, (2.7) implies

$$
\lim _{m \rightarrow \infty}\left\|P_{n} K_{m}-P_{n} K\right\| \leqq\left\|P_{n}\right\| \lim _{m \rightarrow \infty}\left\|K-K_{m}\right\|=0 .
$$

This implies that, for each $n, \lim _{m \rightarrow \infty}\left\|K-P_{n} K_{m}\right\|=\left\|K-P_{n} K\right\|$. The result (2.9) follows by letting $n \rightarrow \infty$ and employing (2.8). The stronger result (2.10) follows immediately from the relation $\left\|K-P_{n} K_{m}\right\| \leqq\left\|K-P_{n} K\right\|+\left\|P_{n}\right\|\left\|K-K_{m}\right\|$ using (2.7), (2.8), and the uniform boundedness of $\left\{\left\|P_{n}\right\|\right\}$. When (2.11) holds, Banach's theorem [9, p. 172] implies $\left(\lambda I-P_{n} K_{m}\right)^{-1}$ exists. The bound (2.12) follows from the identity

$$
\left(\lambda I-P_{n} K_{m}\right)\left(x^{*}-x_{n m}\right)=\lambda\left(x^{*}-P_{n} x^{*}\right)+P_{n}\left(K-K_{m}\right) x^{*} .
$$

This completes the proof.

When Galerkin's method is applied, approximations are generally also involved in evaluating the integrals with respect to $s$ in (2.5). Let $\left\{Q_{k}\right\}$ be a sequence of quadrature rules such that, for each $f \in C[a, b]$,

$$
Q_{k}(f) \rightarrow \int_{a}^{b} w(t) f(t) d t \quad \text { as } k \rightarrow \infty
$$

Define an approximation $P_{n k}$ to the Fourier projection operator $P_{n}$ by

$$
\left(P_{n k} f\right)(s)=\sum_{i} Q_{k}\left(u_{i} f\right) u_{i}(s)
$$


Then (2.13) implies

$$
P_{n k} f \rightarrow P_{n} f \quad \text { as } k \rightarrow \infty, \text { for each } f \in C[a, b] .
$$

When approximate operators $K_{m}$ and $P_{n k}$ are used, (1.3) is replaced by

$$
\left(\lambda I-P_{n k} K_{m}\right) x=P_{n k} y .
$$

TheOREM 2.2. Conditions (2.7), (2.8) and (2.15) imply

$$
\lim _{n \rightarrow \infty} \lim _{k, m \rightarrow \infty}\left\|K-P_{n k} K_{m}\right\|=0 \text {. }
$$

For all $n, k, m$ such that

$$
\left\|K-P_{n k} K_{m}\right\|\left\|(\lambda I-K)^{-1}\right\|<1,
$$

a unique solution $\tilde{x}$ of (2.16) exists, and

$$
\left\|x^{*}-\tilde{x}\right\| \leqq\left\|\left(\lambda I-P_{n k} K_{m}\right)^{-1}\right\|
$$

$$
\begin{aligned}
\cdot\left\{|\lambda|\left(1+\left\|P_{n}\right\|\right) \operatorname{dist}\left(x^{*} ; S_{n}\right)+\right. & \left\|P_{n k}\right\|\left\|\left(K-K_{m}\right) x^{*}\right\| \\
& \left.+|\lambda|\left\|\left(P_{n}-P_{n k}\right) x^{*}\right\|\right\} .
\end{aligned}
$$

Proof. We first show that, for each $n$,

$$
\lim _{k, m \rightarrow \infty}\left\|K-P_{n k} K_{m}\right\|=\left\|K-P_{n} K\right\| .
$$

Let $n$ be given and fixed. To establish (2.20), it is sufficient to show that

$$
\lim _{k, m \rightarrow \infty}\left\|P_{n} K-P_{n k} K_{m}\right\|=0 .
$$

The compactness of $K$ and (2.15) imply

$$
\left\|P_{n k} K-P_{n} K\right\| \rightarrow 0 \text { as } k \rightarrow \infty .
$$

(2.15) also implies $\left\{P_{n k}\right\}$ is uniformly bounded over $k$. That is, there exists a number $M_{n}$ depending only on $n$, such that $\left\|P_{n k}\right\| \leqq M_{n}$ for all $k$. Now

$$
\begin{aligned}
\left\|P_{n} K-P_{n k} K_{m}\right\| & \leqq\left\|P_{n} K-P_{n k} K\right\|+\left\|P_{n k} K-P_{n k} K_{m}\right\| \\
& \leqq\left\|P_{n} K-P_{n k} K\right\|+M_{n}\left\|K-K_{m}\right\|
\end{aligned}
$$

The result (2.20) now follows using (2.21) and (2.7). Finally, if we take the limit as $n \rightarrow \infty$ of each side of (2.20) and employ (2.8), we obtain (2.17).

The bound (2.19) is derived from the identity

$$
\left(\lambda I-P_{n k} K_{m}\right)\left(x^{*}-\tilde{x}\right)=\lambda\left(x^{*}-P_{n} x^{*}\right)+P_{n k}\left(K-K_{m}\right) x^{*}+\lambda\left(P_{n}-P_{n k}\right) x^{*} .
$$

Although (2.12) and (2.19) do not generally provide computable error bounds, they are useful in practice for obtaining order of convergence estimates. Examples given in the next section illustrate such usage. 
It is interesting to note that the right-hand side $y$ of (1.1) appears explicitly in neither (2.12) nor (2.19). It is the smoothness of $x^{*}=\lambda^{-1}\left(y+K x^{*}\right)$, not of $y$ or $K x^{*}$ individually, which determines the rate of convergence of the solution of (2.6) or (2.16).

3. Quadrature Rules and Examples. It is not necessary that (2.7) hold in order to successfully use a projection method. However, useful operator approximations $\left\{K_{m}\right\}$ which converge uniformly to $K$ can be easily constructed in many cases.

The approximations given here are similar to those suggested in [1]. In [1], integrals of the form $\int_{a}^{b} k(s, t) u(t) d t$ are approximated by writing $k_{s}(t)=k(s, t)$ in the form $k_{s}(t)=r_{s}(t) h_{s}(t)$ where $r_{s}(t)$ is smooth and $h_{s}(t)$ can be integrated analytically. The function $r_{s}(t) u(t)$ is then replaced by an approximation $g_{s}(t)$ which is of simple form, e.g. a piecewise polynomial. If $h_{s}(t)$ has been chosen properly, the product $h_{s}(t) g_{s}(t)$ can be integrated analytically.

The functions $u_{j}(t)$ used in (1.7) are generally chosen to have simple form. Hence, we modify the technique above so that only $r_{s}$, not $r_{s} u_{i}$, is replaced by an approximation. More generally, we have the following:

THEOREM 3.1. Suppose

$$
k(s, t)=\sum_{p=1}^{q} r_{p}(s, t) h_{p}(s, t)
$$

where, for each $p$,

$$
\begin{gathered}
r_{p} \in C([a, b] \times[a, b]), \\
\eta_{p}=\sup _{a \leqq s \leqq b} \int_{a}^{b}\left|h_{p}(s, t)\right| d t<\infty,
\end{gathered}
$$

and

$$
\sup _{|s-t| \leqq \delta} \int_{a}^{b}\left|h_{p}(s, \tau)-h_{p}(t, \tau)\right| d \tau \rightarrow 0 \quad \text { as } \delta \rightarrow 0 .
$$

Let $\left\{V_{m}\right\}$ be a sequence of bounded linear maps from $C[a, b]$ onto the space of bounded integrable functions on $[a, b]$. For each $m$, define $K_{m}$ by

$$
\left(K_{m} f\right)(s)=\sum_{p} \int_{a}^{b} V_{m}\left[r_{p}(s, t)\right] h_{p}(s, t) f(t) d t, \quad f \in C[a, b],
$$

where it is assumed $V_{m}$ is applied to $r_{p}$ as a function of $t$. Then

$$
K_{m}: C[a, b] \rightarrow C[a, b]
$$

and

$$
\left\|K-K_{m}\right\| \leqq \alpha_{m} \sum_{p} \eta_{p}
$$

where

$$
\alpha_{m}=\max _{p} \sup _{s, t}\left|r_{p}(s, t)-V_{m}\left[r_{p}(s, t)\right]\right| .
$$

Hence, if $\alpha_{m} \rightarrow 0$ as $m \rightarrow \infty$, then $\left\|K-K_{m}\right\| \rightarrow 0$.

Then

Proof. Let $\beta=\max _{p} \sup _{s, t}\left|r_{p}(s, t)\right|$. Fix $m$, and let $g \in C[a, b]$, and $s, t \in[a, b]$. 


$$
\begin{aligned}
\left|\left(K_{m} g\right)(s)-\left(K_{m} g\right)(t)\right| \leqq & \|g\| \sum_{p} \int_{a}^{b}\{ \\
& \left\{\left|V_{m}\left[r_{p}(s, \tau)\right]\left[h_{p}(s, \tau)-h_{p}(t, \tau)\right]\right|\right. \\
& \left.+\left|V_{m}\left[r_{p}(s, \tau)-r_{p}(t, \tau)\right] h_{p}(t, \tau)\right|\right\} d \tau \\
\leqq & \|g\|\left\|V_{m}\right\|\left\{\beta \sum_{p} \int_{a}^{b}\left|h_{p}(s, \tau)-h_{p}(t, \tau)\right| d \tau\right. \\
& \left.\quad+\max _{p} \sup _{\tau}\left|r_{p}(s, \tau)-r_{p}(t, \tau)\right| \sum_{p} \eta_{p}\right\}
\end{aligned}
$$

Thus, (3.6) follows from (3.2)-(3.4). To obtain (3.7), note that, for each $g \in C[a, b]$, $\left\|K g-K_{m} g\right\|=\sup _{s}\left|\int_{a}^{b} \sum_{p}\left[r_{p}(s, t)-V_{m}\left(r_{p}(s, t)\right)\right] h_{p}(s, t) g(t) d t\right| \leqq\|g\| \alpha_{m} \sum_{p} \eta_{p}$.

This completes the proof.

Collocation Example. Suppose (1.1) is solved approximately by collocation using a cubic spline subspace. Let $\left\{\pi_{n}\right\}$ be a sequence of partitions of $[a, b], \pi_{n}: a=t_{0 n}<$ $t_{1 n}<\cdots<t_{n n}=b$ such that $\left|\pi_{n}\right|=\max _{i}\left(t_{i n}-t_{i-1, n}\right) \rightarrow 0$ as $n \rightarrow \infty$. For each $n$, let $S_{n}$ denote the subspace of cubic splines with knots on $\pi_{n}$, and let $P_{n}$ be the interpolation projection onto $S_{n}$ with interpolating points on $\pi_{n}$ and at $t_{0 n}=\left(t_{1 n}+t_{0 n}\right) / 2$, $\tilde{t}_{n n}=\left(t_{n n}+t_{n-1, n}\right) / 2$. Assume the partitions $\pi_{n}$ have uniformly bounded mesh ratios $q_{n}=\left|\pi_{n}\right| / \min _{i}\left(t_{i n}-t_{i-1, n}\right)$. Then the projections $P_{n}$ converge pointwise to the identity [3] and are thus uniformly bounded.

Suppose the kernel function $k(s, t)$ can be expressed in the form (3.1) where, for each $p, r_{p} \in C^{(2)}[a, b]$ as a function of $t$, and $h_{p}$ satisfies (3.3) and (3.4). Let $V_{m}$ denote the interpolation projection onto the space of linear splines (broken lines) with knots on $\pi_{m}$. Then [4] $\alpha_{m}=O\left(\left|\pi_{m}\right|^{2}\right)$, so (3.7) implies $\left\|K-K_{m}\right\|=O\left(\left|\pi_{m}\right|^{2}\right)$. Moreover, (2.8) holds as can be seen by applying Theorem 4.1 of [13]. Theorem 2.1 now applies. Thus, a unique solution of (2.6) exists for all suificiently large $m$ and $n$. If $x^{*} \in C^{(k)}[a, b], 0 \leqq k \leqq 4$, then [4] dist $\left(x^{*} ; S_{n}\right)=O\left(\left|\pi_{n}\right|^{k}\right)$, so from (2.12), we obtain

$$
\left\|x^{*}-x_{n m}\right\|=O\left(\left|\pi_{n}\right|^{k}\right)+O\left(\left|\pi_{m}\right|^{2}\right) .
$$

Galerkin Example. Let $P_{n}$ denote the Fourier-Chebyshev projection operator onto the space $\mathbf{P}_{n}$ of polynomials of degree not greater than $n$. For each $f \in C[a, b]$, $P_{n} f$ is given by

$$
\begin{aligned}
\left(P_{n} f\right)(s) & =\sum_{j=0}^{n} I_{j}(f) T_{j}(s), \\
I_{i}(f) & =\frac{2}{\pi} \int_{-1}^{1}\left(1-t^{2}\right)^{-1 / 2} T_{j}(t) f(t) d t,
\end{aligned}
$$

where $T_{j}(s)$ denotes the Chebyshev polynomial of degree $j$, and $\sum^{\prime}$ denotes the first term in the summation is to be halved. Suppose $(a, b)=(-1,1)$ and that $(1.1)$ is to be solved approximately using Galerkin's method with the projection $P_{n}$ in (3.9).

Using the substitution $t=\cos \theta$, the integrals $I_{j}(f)$ can be expressed as

$$
I_{i}(f)=\frac{2}{\pi} \int_{0}^{\pi} \cos (j \theta) f(\cos \theta) d \theta
$$


If each $I_{i}$ is approximated using the trapezoidal rule with spacing $h=\pi / k, k \geqq n$, (3.10) is replaced by

$$
\tilde{I}_{j}(f)=\frac{2}{k} \sum_{m=0}^{k} \prime \prime \cos \left(j \theta_{m}\right) f\left(\cos \theta_{m}\right), \quad \theta_{m}=m \pi / k,
$$

where $\sum^{\prime \prime}$ denotes the first and last summands are to be halved. The $\tilde{I}_{i}(f)$ are $[7$, p. 31$]$ coefficients in the discrete least squares Chebyshev expansion

$$
\left[\tilde{P}_{n k} f\right](s)=\sum_{i=0}^{n}, \tilde{I}_{j}(f) T_{i}(s) .
$$

Thus, $\tilde{P}_{n k}$ is itself a projection operator onto $\mathrm{P}_{n}$. As a consequence, use of the trapezoidal rule to evaluate the integrals involving $w(s)=\left(1-s^{2}\right)^{-1 / 2}$ in (2.5) implies that a discrete Galerkin method is actually being used to solve (1.1) approximately. Hence, (2.12) can be used instead of (2.19) to analyze convergence.

When $k=n$, the projection operator $\tilde{P}_{n k}$ becomes interpolation onto $\mathbf{P}_{n}$ at the points $\cos \theta_{m}$. In this case [6], $\left\|\tilde{P}_{n n}\right\|=O(\ln n)$. Suppose $x^{*(4)}$ exists and is bounded. Then by Jackson's Theorem [11], dist $\left(x^{*} ; \mathbf{P}_{n}\right)=O\left(n^{-4}\right)$. Thus, (2.12) becomes

$$
\left\|x^{*}-x_{n m}\right\|=O(\ln n)\left[O\left(n^{-4}\right)+O\left(\left\|\left(K-K_{m}\right) x^{*}\right\|\right)\right] .
$$

Instead of (3.11), suppose the Gauss-Chebyshev quadrature formula

$$
\int_{-1}^{1}\left(1-t^{2}\right)^{-1 / 2} g(t) d t=\frac{\pi}{k} \sum_{j=1}^{k} g\left(\cos \xi_{j}\right)+E(g), \quad \xi_{j}=\left(j-\frac{1}{2}\right) \pi / k,
$$

with $k \geqq n+1$ is used to approximate the integrals in (3.9). The resulting approximation to $P_{n}$ is given by

$$
\left(P_{n k} f\right)(s)=\sum_{j=0}^{n}, \hat{I}_{j}(f) T_{i}(s), \quad \hat{I}_{i}(f)=\frac{2}{k} \sum_{m=1}^{k} \cos \left(j \xi_{m}\right) f\left(\cos \xi_{m}\right) .
$$

$P_{n k}$ defines another discrete least squares Chebyshev expansion [7, p. 32]. Thus we are led to a second discrete Galerkin method. Rather than use (2.12) to analyze this method, we illustrate the use of (2.19).

For any function $f$, the approximation $P_{n k} f$ differs from $P_{n} f$ by no more than

$$
\left\|P_{n} f-P_{n k} f\right\| \leqq \frac{2}{\pi} \sum_{i=0}^{n}\left|E\left(T_{i} f\right)\right|\left\|T_{i}\right\|=\frac{2}{\pi} \sum_{j}^{\prime}\left|E\left(T_{i} f\right)\right| .
$$

Again assume $x^{*(4)}$ exists and is bounded. Then for each $j$, dist $\left(x^{*} T_{i} ; \mathbf{P}_{n+j}\right)=O\left(n^{-4}\right)$, so [5, Section 4.8] $\left|E\left(x^{*} T_{i}\right)\right|=O\left(n^{-4}\right)$. Thus, (3.15) implies $\left\|P_{n} x^{*}-P_{n k} x^{*}\right\|=O\left(n^{-3}\right)$. A crude bound on $\left\|P_{n k}\right\|$ is given by $\left\|P_{n k}\right\| \leqq \sum_{i}^{\prime}\left\|\hat{I}_{i}\right\|\left\|T_{i}\right\|=2 n+1$, while [11] $\left\|P_{n}\right\|=O(\ln n)$. Thus, (2.19) implies $\left\|x^{*}-\tilde{x}\right\|=O\left(n^{-3}\right)+O\left(n\left\|\left(K-K_{m}\right) x^{*}\right\|\right)$.

4. Finite Rank Operator Methods. Suppose the solution $x^{*}$ of (1.2) is approximated by the solution $\tilde{x}_{n}$ of an equation

$$
\left(\lambda I-K_{n}\right) \tilde{x}_{n}=y,
$$

where $K_{n}$ is an operator of finite rank. We refer to any such approximation method as a finite rank operator method. In this section, we will show the relation between finite rank operator methods and projection methods. We then apply the analysis of Section 2 to certain of these methods. 
Any bounded linear operator $K_{n}$ of finite rank defined on $C[a, b]$ can be expressed in the form

$$
\left(K_{n} f\right)(s)=\sum_{i=1}^{n} \mu_{i}(f) u_{i}(s),
$$

where the $\mu_{i}$ are bounded linear functionals on $C[a, b]$ and $\left\{u_{i}\right\}$ spans the range of $K_{n}$. If the approximating equation (4.1) has a unique solution $\tilde{x}_{n}(s)$, the solution must satisfy

$$
\lambda \tilde{x}_{n}(s)-\sum_{i} c_{j} u_{i}(s)=y(s), \quad c_{i}=\mu_{i}\left(\tilde{x}_{n}\right) .
$$

Hence, $\tilde{x}_{n}(s)$ has the form

$$
\tilde{x}_{n}(s)=\lambda^{-1}\left(y(s)+\sum_{i} c_{i} u_{i}(s)\right)
$$

The $c_{i}$ satisfy the linear system

$$
\lambda c_{i}-\sum_{j} c_{i} \mu_{i}\left(u_{i}\right)=\mu_{i}(y), \quad i=1, \cdots, n,
$$

obtained by applying $\mu_{i}$ to each side of (4.3). In fact, $\tilde{x}_{n}$ given by (4.4) is a solution of (4.1) if and only if the $c_{i}$ satisfy (4.5). Thus, (4.1) has a unique solution if and only if (4.5) does. The solvability of (4.5) does not depend on whether or not $\left\{\mu_{i}\right\}$ or $\left\{u_{i}\right\}$ is linearly independent.

Now suppose $P_{n}$ is a projection operator defined by (1.5) and $K_{n}$ is the operator defined by (4.2), where the functionals $\mu_{i}$ are identical with those in (1.5). If the operator $K_{n}=K_{m}$ defined in (4.2) is used in (2.6), the solution $x_{n n}$ of (2.6) is related to the solution of (4.1) by

$$
x_{n n}=P_{n} \tilde{x}_{n} .
$$

To see this, let $\left\{y_{i}\right\}$ be a basis of the range of $P_{n}$ satisfying (1.4). In solving (2.6), the coefficients $d_{i}$ in the expansion $x_{n n}(s)=\sum_{i} d_{i} y_{i}(s)$ are determined from the linear system

$$
\sum_{i}\left[\lambda \delta_{i i}-\mu_{i}\left(K_{n} y_{i}\right)\right] d_{i}=\mu_{i}(y), \quad i=1, \cdots, n .
$$

But (4.2) and (1.4) imply $K_{n} y_{j}=\sum_{i} \mu_{i}\left(y_{i}\right) u_{i}=u_{i}$. Hence, the system (4.7) is identical with (4.5). Since $c_{i}=\mu_{j}\left(\tilde{x}_{n}\right)$ and $d_{j}=\mu_{j}\left(x_{n n}\right)$, this implies $\mu_{j}\left(\tilde{x}_{n}\right)=\mu_{j}\left(x_{n n}\right), j=$ $1, \cdots, n$. Hence, $P_{n} \tilde{x}_{n}=P_{n} x_{n n}$. But $P_{n} x_{n n}=x_{n n}$, so (4.6) must hold.

The Nyström method and the method of collocation illustrate the relation (4.6). The Nyström method ([12], [2]) is derived by replacing the integral in (1.1) by a quadrature rule

$$
\int_{a}^{b} g(t) d t \doteq \sum_{i=1}^{n} w_{i} g\left(t_{i}\right)
$$

The Nyström method is thus a finite rank operator method where the functionals $\mu_{i}$ in (4.2) are given by point evaluation at $t_{i}$ and $u_{i}(s)=w_{i} k\left(s, t_{i}\right)$. More generally [1], if $k(s, t)$ is expressed in the form (3.1), a product quadrature rule

$$
\int_{a}^{b} f(t) g(t) d t \doteq \sum_{i=1}^{n} w_{i} g\left(t_{i}\right)
$$


might be used rather than (4.8). In this case the $u_{i}(s)$ have the form $u_{i}(s)=$ $\sum_{p} w_{i p}(s) r_{p}\left(s, t_{i}\right)$.

Suppose the Nyström method is applied to (1.1), and an interpolate $\hat{x}_{n}=P_{n} \tilde{x}_{n}$ of the resulting approximate solution $\tilde{x}_{n}$ is formed such that $\hat{x}_{n}\left(t_{i}\right)=\tilde{x}_{n}\left(t_{i}\right), i=1, \cdots, n$. Then (4.6) implies that $\hat{x}_{n}$ is the same function found by solving (1.1) approximately using collocation at the points $\left\{t_{i}\right\}$ with the integrals evaluated using the same quadrature rule (4.8) or (4.9) used in determining $\tilde{x}_{n}$. This relation between the Nyström method and collocation has been noted before ([9, Section XIV. 4], [14]). The work in Section 2 provides a means of analyzing the error in the approximate solution found.

A second well-known finite rank operator method is the degenerate kernel method. In this method [10], $k(s, t)$ is replaced by a degenerate kernel

$$
k_{n}(s, t)=\sum_{i=1}^{n} \alpha_{i}(s) \beta_{i}(t)
$$

The functionals $\mu_{i}$ in (4.2) are given by

$$
\mu_{i}(f)=\int_{a}^{b} f(t) \beta_{i}(t) d t
$$

while the $u_{i}$ are given by $u_{i}(s)=\alpha_{i}(s)$, for each $i$.

One means of obtaining a kernel (4.10) which approximates $k(s, t)$ is to use $k_{n}(s, t)=P_{n} k(s, t)$, where $P_{n}$ is a projection operator applied to $k(s, t)$ as a function of $s$. The operator $K_{n}$ in (4.1) now has the form

$$
K_{n}=P_{n} K \text {. }
$$

The solution $\tilde{x}_{n}$ of (4.1) satisfies

$$
\tilde{x}_{n}=\lambda^{-1}\left(y+z_{n}\right), \quad z_{n}=P_{n} K \tilde{x}_{n},
$$

where $z_{n}$ can be found as the solution of

$$
\left(\lambda I-P_{n} K\right) z_{n}=P_{n} K y .
$$

Thus, application of the degenerate kernel method with an approximation operator of the form (4.12) is equivalent to solving the regularized equation [9, p. 552]

$$
(\lambda I-K) z=K y,
$$

using the method of projections, then defining $\tilde{x}_{n}$ by (4.13). As we see below, this equivalence permits us to study the error in $\tilde{x}_{n}$ using the analysis of Section 2 .

Note that the solution $x^{*}$ of (1.2) satisfies $(\lambda I-K) K x^{*}=K y$. Comparing this with (4.15), we see that the solution $z^{*}$ of (4.15) satisfies $z^{*}=K x^{*}$. Moreover, (4.13) and (4.12) imply $z_{n}=K_{n} \tilde{x}_{n}$. These relations, together with (1.2), (4.1) yield

$$
z^{*}-z_{n}=K x^{*}-K_{n} \tilde{x}_{n}=\left(\lambda x^{*}-y\right)-\left(\lambda \tilde{x}_{n}-y\right)=\lambda\left(x^{*}-\tilde{x}_{n}\right) .
$$

Using (4.14) then (4.15), we have

$$
\begin{aligned}
\left(\lambda I-P_{n} K\right)\left(z^{*}-z_{n}\right) & =\lambda z^{*}-P_{n} K z^{*}-P_{n} K y \\
& =\lambda z^{*}-P_{n}\left(\lambda z^{*}-K y\right)-P_{n} K y=\lambda\left(I-P_{n}\right) z^{*},
\end{aligned}
$$

so (4.16) implies $\left(\lambda I-P_{n} K\right)\left(x^{*}-\tilde{x}_{n}\right)=\left(I-P_{n}\right) z^{*}$. Hence, if $S_{n}=$ range of $P_{n}$, the error in $\tilde{x}_{n}$ is bounded by 


$$
\left\|x^{*}-x_{n}\right\| \leqq\left\|\left(\lambda I-P_{n} K\right)^{-1}\right\|\left(1+\left\|P_{n}\right\|\right) \operatorname{dist}\left(z^{*} ; S_{n}\right) .
$$

By comparison, we note that if the same projection operator is used in (1.3), the resulting approximation $x_{n}$ satisfies

$$
\begin{aligned}
\left(\lambda I-P_{n} K\right)\left(x^{*}-x_{n}\right) & =\lambda x^{*}-P_{n} K x^{*}-P_{n} y \\
& =\lambda x^{*}-P_{n}\left(\lambda x^{*}-y\right)-P_{n} y=\lambda\left(I-P_{n}\right) x^{*},
\end{aligned}
$$

so

$$
|| x^{*}-x_{n}\|\leqq\|\left(\lambda I-P_{n} K\right)^{-1}|||\lambda|\left(1+\left\|P_{n}\right\|\right) \operatorname{dist}\left(x^{*} ; S_{n}\right) .
$$

Comparing (4.17) and (4.18), one would expect $\tilde{x}_{n}$ to be a better approximation to $x^{*}$ than $x_{n}$ whenever $z^{*}=\lambda x^{*}-y$ can be better approximated than $x^{*}$ by functions in $S_{n}$.

In practice, approximate operators $K_{m}$ and $P_{n k}$ might be used in place of $K$ and $P_{n}$ when solving (4.14) numerically. If, instead of (4.14), an approximate equation

$$
\left(\lambda I-P_{n k} K_{m}\right) \tilde{z}=P_{n k} K y
$$

is solved, the situation is analogous to (2.16) and an error bound for $z^{*}-\tilde{z}$ can be found using (2.19). Generally, however, one would probably also replace $K$ on the right-hand side of (4.19) by the approximate operator $K_{m}$. Thus, the equations

$$
\left(\lambda I-P_{n k} K_{m}\right) \tilde{z}=P_{n k} K_{m} y, \quad \tilde{x}=\lambda^{-1}(y+\tilde{z})
$$

would be solved to obtain an approximation $\tilde{x}$ to $x^{*}$. Then

$$
\left(\lambda I-P_{n k} K_{m}\right)\left(x^{*}-\tilde{x}\right)=\left(z-P_{n} z^{*}\right)+\left(P_{n}-P_{n k}\right) z^{*}+P_{n k}\left(K-K_{m}\right) x^{*},
$$

so analogous to (2.19), we have

$$
\begin{aligned}
\left\|x^{*}-\tilde{x}\right\| & \leqq\left\|\left(\lambda I-P_{n k} K_{m}\right)^{-1}\right\| \\
\cdot & \left\{\left(1+\left\|P_{n}\right\|\right) \operatorname{dist}\left(z^{*} ; S_{n}\right)+\left\|P_{n k}\right\|\left\|\left(K-K_{m}\right) x^{*}\right\|+\left\|\left(P_{n}-P_{n k}\right) z^{*}\right\|\right\} .
\end{aligned}
$$

Computer Science Department

Washington State University

Pullman, Washington 99163

1. K. E. AtKInson, "The numerical solution of Fredholm integral equations of the second kind," SIAM J. Numer. Anal., v. 4, 1967, pp. 337-348. MR 36 \#358.

2. K. E. AtKinson, "A survey of numerical methods for the solution of Fredholm integral equations of the second kind," 1971 Fall National Meeting of SIAM, Madison, Wisconsin (To appear.)

3. C. R. DEBoor, The Method of Projections as Applied to the Numerical Solution of Two Point Boundary Value Problems Using Cubic Splines, Doctoral Thesis, University of Michigan, Ann Arbor, Mich., 1966.

4. C. R. DEBOOR, "On uniform approximation by splines," J. Approximation Theory, v. 1, 1968, pp. 219-235. MR 39 \#1866.

5. P. J. Davis \& P. Rabinowitz, Numerical Integration, Blaisdell, Waltham, Mass., 1967. MR 35 \# 2482.

6. H. Ehlich \& K. Zeller, "Auswertung der Normen von Interpolationsoperatoren," Math. Ann., v. 164, 1966, pp. 105-112. MR 33\#3005.

7. L. Fox \& I. B. Parker, Chebyshev Polynomials in Numerical Analysis, Oxford Univ. Press, London, 1968. MR 37 \#3733.

8. Y. IKEBE, "The Galerkin method for numerical solution of Fredholm integral equations of the second kind," SIAM Rev., v. 14, 1972, pp. 465-491. 
9. L. V. KANTOROvič \& G. P. AkILov, Functional Analysis in Normed Spaces, Fizmatgiz, Moscow, 1959; English transl., Internat. Series of Monographs in Pure and Appl. Math., vol. 46, Macmillan, New York, 1964. MR 22 \#9837; MR 35 \#4699.

10. L. V. Kantorovič \& V. I. Krylov, Approximate Methods of Higher Analysis, 3rd ed., GITTL, Moscow, 1950; English transl., Interscience, New York; Noordhoff, Groningen, 1958. MR 13, 77; MR 27 \#4338.

11. I. P. Natanson, Constructive Function Theory, GITTL, Moscow, 1949; English transl., Vol. I, Ungar, New York, 1964; Vols. II, III, 1965. MR 11, 591; MR 33 \#4529a, b, c. 12. E. J. NYSTRöM, "Über die praktische Auflösung von Integralgleichungen mit Anwendungen auf Randwertaufgaben," Acta. Math., v. 54, 1930, pp. 185-204.

13. J. L. Phillips, "The use of collocation as a projection method for solving linear operator equations," SIAM J. Numer. Anal., v. 9, 1972, pp. 14-28.

14. P. M. Prenter, "A method of collocation for the numerical solution of integral equations of the second kind." (To appear.) 